\title{
Current use of combined hormonal contraception is associated with glucose metabolism disorders in perimenopausal women
}

\author{
Maria-Elina Mosorin', Annina Haverinen², Meri-Maija Ollila', Tanja Nordström, ${ }^{3,5}$, Jari Jokelainen ${ }^{3,5}$, \\ Sirkka Keinänen-Kiukaanniemi' ${ }^{3,4}$, Katri Puukka6 , Aimo Ruokonen' ${ }^{6}$, Juha Auvinen ${ }^{3,4}$, Terhi Piltonen', \\ Laure Morin-Papunen ${ }^{1}$ and Juha S Tapanainen ${ }^{1,2}$
}

'Department of Obstetrics and Gynecology, Oulu University Hospital, University of Oulu and Medical Research Center, and PEDEGO Research Unit (Research Unit for Pediatrics, Dermatology, Clinical Genetics, Obstetrics and Gynecology), Oulu, Finland, ${ }^{2}$ Department of Obstetrics and Gynecology, University of Helsinki and Helsinki University Hospital, Helsinki, Finland, ${ }^{3}$ Center for Life Course Health Research, University of Oulu, Oulu, Finland, ${ }^{4}$ Unit of General Practice, Oulu University Hospital, Oulu, Finland, ${ }^{5}$ Medical Research Center Oulu, Oulu University Hospital, University of Oulu, Oulu, Finland, and ${ }^{6}$ Nordlab Oulu, Medical Research Center Oulu, Oulu University Hospital and Department of Clinical Chemistry, University of Oulu, Oulu, Finland

Correspondence should be addressed to J S Tapanainen Email

juha.tapanainen@helsinki.fi

\begin{abstract}
Objective: The use of combined hormonal contraceptives (CHCs) worsens glucose tolerance, but the risk for glucose metabolism disorders remains controversial.

Design: The study is a prospective longitudinal population-based cohort study.

Methods: The study was based on a cohort population that comprised 1879 women born in 1966 . At age 46, the women answered a questionnaire on contraceptive use and underwent an oral glucose tolerance test. Glucose metabolism indices were evaluated in current CHC $(n=153)$, progestin-only contraceptive (POC, $n=842)$, and nonhormonal contraceptive users $(n=884)$.

Results: In the entire study population, current $\mathrm{CHC}$ use was significantly associated with prediabetes (OR: 2.0, 95\% $\mathrm{Cl}: 1.3-3.2$ ) and type 2 diabetes (OR: 3.3, 95\% Cl: 1.1-9.7) compared to non-hormonal contraceptive use. After 5 years of use, the prediabetes risk increased 2.2 -fold ( $95 \% \mathrm{Cl}: 1.3-3.7)$ and type 2 diabetes risk increased 4.5 -fold ( $95 \% \mathrm{Cl}$ : 1.5-13.5). Compared with the current POC use, current $\mathrm{CHC}$ use was significantly associated with prediabetes (OR: 1.9, 95\% Cl: 1.2-3.0). Current POC use was not associated with any glucose metabolism disorders. The results prevailed after adjusting for BMI and socioeconomic status.

Conclusions: $\mathrm{CHC}$ use in perimenopausal women was associated with a significantly increased risk of glucose metabolism disorders. This association should be considered in women with increased metabolic risk.
\end{abstract}

\section{Introduction}

Early-generation combined hormonal contraceptives (CHCs) have been shown to negatively impact glucose metabolism, which results in impaired fasting glucose and glucose intolerance $(1,2,3)$. In the past, these preparations contained relatively high doses of ethinyl estradiol (EE; $50-150 \mu \mathrm{g}$ ) and androgenic progestins (4), whereas modern preparations consist of low-dose EE (20-30 $\mu \mathrm{g})$ and less androgenic or even antiandrogenic progestins (5). 
One of our previous studies in young, normal-weight women demonstrated that continuous use of CHCs for 9 weeks, regardless of administration route, worsened glucose tolerance and induced chronic inflammation (6). These results are consistent with those of most other studies $(7,8,9)$ but not all (10). A Cochrane review indicated that $\mathrm{CHC}$ use had only a limited effect on glucose metabolism in healthy, normal-weight women (11). However, oral glucose tolerance tests (OGTTs) were not performed in all studies, and only a few of them considered BMI. Progestin-only contraceptives (POCs) have been less studied, but they have been associated with minimal $(12,13,14,15,16)$ or no alterations $(8,17,18)$ in glucose metabolism depending on the preparation.

Studies on associations between the risk of type 2 diabetes (T2DM) and CHC use demonstrate conflicting results and are difficult to compare due to differences in study designs, study populations, and hormonal contraceptives (11). However, a moderately elevated risk of diabetes has been observed among premenopausal Chinese women using CHCs (19), and a large prospective population-based Swedish study demonstrated a significantly increased risk of prediabetes (preDM) in current $\mathrm{CHC}$ users over 36 years of age (20). The use of CHCs is often long-term, and CHCs are increasingly being prescribed for older women $(21,22)$, as their use has been considered to be safe up to menopause in nonsmoking, healthy women with no known risk factors of cardiovascular disease. Thus, currently available data suggest that $\mathrm{CHC}$ use increases the risk of diabetes, but whether these risks translate to overt disease remains unclear.

In the present study, we investigated the effects of CHCs, POCs, and non-hormonal contraceptives on the occurrence of preDM and T2DM in perimenopausal women in a prospective, national population-based follow-up cohort.

\section{Subjects and methods}

\section{Study population}

The study population was derived from the unique, prospective, population-based Northern Finland Birth Cohort 1966 (NFBC1966, http://www.oulu.fi/nfbc), which includes all expected births in 1966 in the two northernmost provinces of Finland $(n=12058)$. Of these, 5889 were female. Enrolment in the database began at the $24 \mathrm{th}^{\mathrm{h}}$ gestational week, and thus far, data has been collected at age 1, 14, 31, and 46 years.
From 2012 to 2014, a large questionnaire on main health issues and an invitation to a clinical examination were sent to 5123 women of the cohort who were 46 years old. A total of 3708 (72.4\%) women responded to the questionnaire, and 3280 of these women (88.5\%) also participated in the clinical examination, which included anthropometric measurements and blood samples. After exclusion of participants as described subsequently, all women who answered the questionnaire on the current use of hormonal and non-hormonal contraceptives and underwent OGTTs ( $n=1879)$ were included in the analyses (Fig. 1). All participants provided informed consent, and the study was approved by the Ethics Committee of the Northern Ostrobothnia District (EETTMK 94/2011).

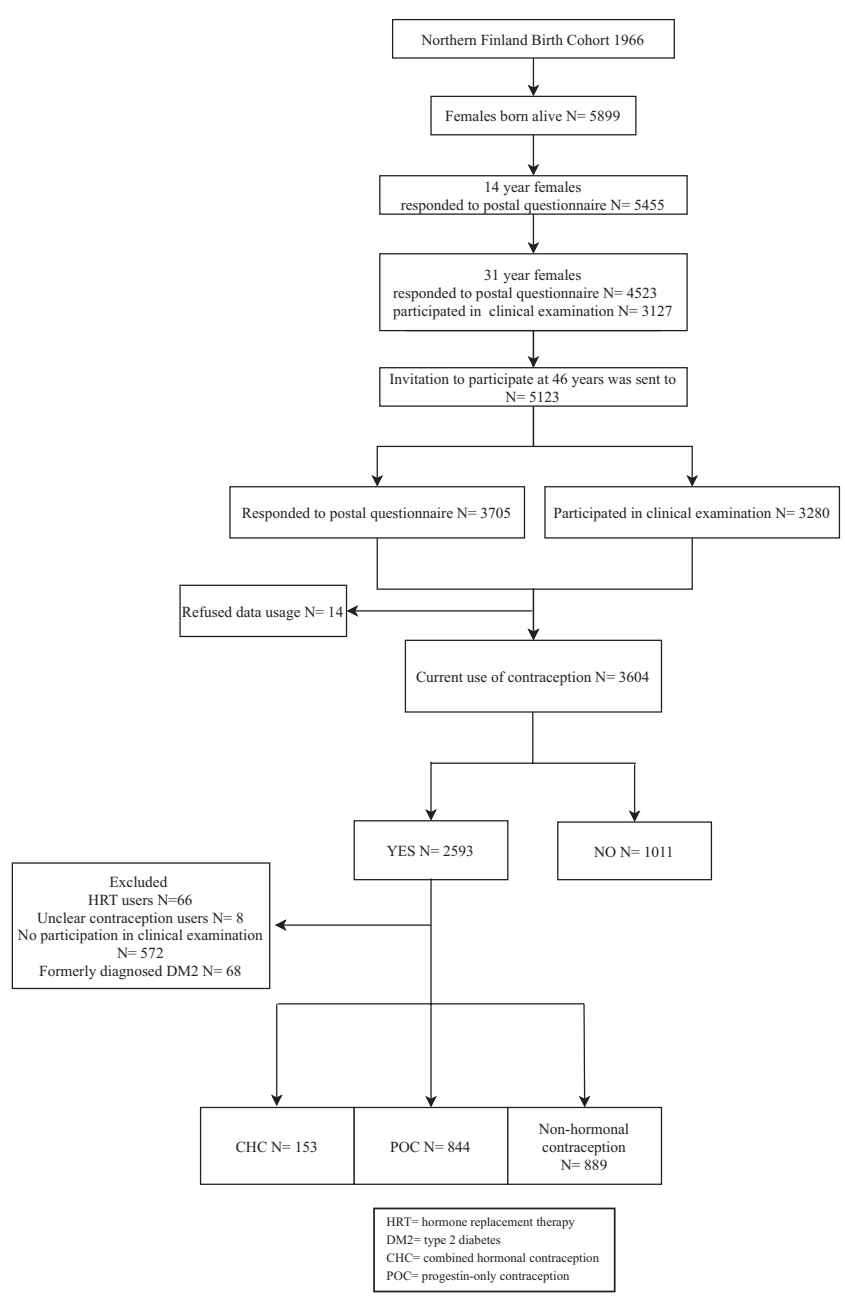

Figure 1

Flow chart of current use of hormonal and non-hormonal contraceptives. 


\section{Current hormonal and non- hormonal contraception}

Women who attended clinical examinations and reported current use of hormonal or non-hormonal contraceptives were divided into three groups: (i) current $\mathrm{CHC}$ (including combined oral contraceptive, vaginal ring, and transdermal patch) users $(n=153)$, (ii) current POC (including progestin-only pill, hormone-releasing intrauterine device, and subdermal capsule) users $(n=842)$, and (iii) current users of non-hormonal contraceptives $(n=884)$ as a reference group, which included all women reporting current use of non-hormonal contraceptives (condom, non-hormonal intrauterine device, or their own or their partner's sterilization). Women who reported non-use of any form of contraception (hormonal or nonhormonal) were excluded from the analyses $(n=1011)$, as this group included more multiparous women, who have a higher risk of metabolic disease as a result of multiple pregnancies and deliveries $(23,24)$. Moreover, women using non-identified hormonal preparations $(n=8)$ or hormonal replacement therapy $(n=66)$ and women with formerly diagnosed T2DM $(n=68)$ were excluded from the analysis. The questionnaire also included a question regarding the length of use of the current form of contraception. A total of 143 (94\%) CHC users, 796 (98\%) POC users, and 512 (58\%) non-hormonal contraceptive users reported the length of use. The data were analyzed for less than 5 years and 5 or greater years of use (Fig. 1).

\section{Anthropometric parameters}

All women who participated in the clinical examination were weighed $(\mathrm{kg})$ using a regularly calibrated digital scale. Height $(\mathrm{cm})$ was measured twice using a standard and calibrated stadiometer. BMI was calculated $\left(\mathrm{kg} / \mathrm{m}^{2}\right)$ using measured height and weight. Waist circumference was measured at the level midway between the lowest rib margin and the iliac crest.

\section{Laboratory methods}

Plasma glucose levels were analyzed with an enzymatic dehydrogenase method, and serum insulin levels were analyzed with a chemiluminometric immunoassay (Advia 1800 and Advia Centaur XP, respectively, Siemens Healthcare Diagnostics, Tarrytown, NY, USA). The samples were analyzed in NordLab Oulu, a testing laboratory (T113) accredited by the Finnish Accreditation Service (FINAS) (EN ISO 15189).

\section{Assessment of glucose metabolism disorders}

A 2-h 75-g OGTT was performed in all 1879 women after an overnight (12-h) fasting period. The exclusion criteria for the OGTT were medication for diabetes or a measured capillary blood glucose level of $>8.0 \mathrm{mmol} / \mathrm{L}$. Both serum insulin and plasma glucose levels were measured at baseline and at 30,60, and 120 min after glucose intake. Glucose tolerance status was classified according to World Health Organization criteria: (i) normal glucose tolerance (NGT) was defined as having a fasting plasma glucose (FPG) level $<6.1 \mathrm{mmol} / \mathrm{L}$ and a 2 -h glucose level $<7.8 \mathrm{mmol} / \mathrm{L}$, (ii) impaired glucose tolerance (IGT) was defined as having an FPG level $<7.0 \mathrm{mmol} / \mathrm{L}$ and a 2-h glucose level of 7.8-11.0 $\mathrm{mmol} / \mathrm{L}$, (iii) impaired fasting glucose (IFG) was defined as having an FPG level 6.1-6.9 mmol/L and a 2-h glucose level $<7.8 \mathrm{mmol} / \mathrm{L}$, and (iv) new T2DM was defined as having an FPG level $\geq 7.0 \mathrm{mmol} / \mathrm{L}$ or a 2 -h glucose level $\geq 11.1 \mathrm{mmol} / \mathrm{L}$. Formerly diagnosed cases of T2DM were identified via responses to postal questionnaires (selfreported diagnoses and use of T2DM medication), and the diagnoses were further confirmed from hospital discharge documents and national drug registers of the Social Insurance Institution of Finland. The presence of IFG or IGT was classified as preDM. Women with type 1 diabetes $(n=151)$ or an undefined diabetes type $(n=76)$ were excluded from the analyses.

Fasting glucose and insulin values were used to calculate fasting indexes: homeostasis model assessment of insulin resistance (HOMA-IR) index (fasting plasma glucose $(\mathrm{FPG}) \times$ fasting serum insulin $(\mathrm{FSI}) / 22.5)$ and the homeostasis model assessment of beta-cell function $($ HOMA2- $\beta)$ index $((20 \times$ FSI $) /($ FPG -3.5$) \times 100)$. Glucose and insulin values in OGTTs were used to calculate insulin and glucose areas under the curve (insulin-AUC and glucose-AUC, respectively) and the Matsuda Index for insulin sensitivity (ISI) $(10,000 \times((\mathrm{FPG} \times \mathrm{FSI}) \times((\mathrm{FPG}+30$ min PG +60 min PG +120 min PG $) / 4) \times((F S I+30 \mathrm{~min}$ SI+60 min SI+120 min SI)/4)) (Matsuda 1999).

\section{Statistical analysis}

The differences between study groups were compared with independent Student $t$-tests for normally distributed variables. Variables with a skewed distribution were log-transformed to obtain a normal distribution. The Bonferroni correction was used because there were multiple t-tests. Binary logistic regression modeling was used to investigate whether the current use of the different hormonal contraceptives (i.e. CHCs and POCs) was 
Table 1 Anthropometric and metabolic parameters in current CHC and POC users compared with non-hormonal contraceptive users. Results are shown as mean \pm S.D.

\begin{tabular}{l}
\hline$n$ \\
$\mathrm{BMI}\left(\mathrm{kg} / \mathrm{m}^{2}\right)$ \\
Waist circumference $(\mathrm{cm})$ \\
Number of deliveries \\
Fasting glucose $(\mathrm{mmol} / \mathrm{L})$ \\
Fasting insulin $(\mathrm{mU} / \mathrm{L})$ \\
Glucose-AUC 0-120 (mmol/L min) \\
Insulin-AUC 0-120 (mU/L min) \\
HOMA-IR \\
Matsuda Index
\end{tabular}

\begin{tabular}{c}
\hline CHCs \\
\hline 153 \\
$26.0 \pm 5.0$ \\
$84.8 \pm 11.6$ \\
$1.52 \pm 1.2$ \\
$5.32 \pm 0.5$ \\
$9.11 \pm 5.3$ \\
$13.91 \pm 3.0$ \\
$127.2 \pm 69.8$ \\
$2.18 \pm 1.4$ \\
$5.04 \pm 2.8$ \\
\hline
\end{tabular}

\begin{tabular}{c}
\hline POCs \\
\hline 842 \\
$26.8 \pm 5.2$ \\
$87.5 \pm 13.0$ \\
$2.11 \pm 1.3$ \\
$5.29 \pm 0.5$ \\
$8.66 \pm 5.6$ \\
$12.96 \pm 2.9$ \\
$118.0 \pm 79.0$ \\
$2.14 \pm 1.8$ \\
$5.93 \pm 3.5$ \\
\hline
\end{tabular}

\begin{tabular}{c}
\hline Non-hormonal \\
\hline 884 \\
$26.2 \pm 5.1$ \\
$86.5 \pm 12.7$ \\
$2.25 \pm 1.4$ \\
$5.30 \pm 0.50$ \\
$8.68 \pm 6.5$ \\
$13.16 \pm 2.8$ \\
$123.1 \pm 80.8$ \\
$2.15 \pm 2.0$ \\
$5.65 \pm 3.0$ \\
\hline
\end{tabular}

\begin{tabular}{c}
\hline $\boldsymbol{p}^{\mathbf{a}}$ \\
\hline NS \\
0.047 \\
$<0.001$ \\
NS \\
NS \\
0.001 \\
NS \\
NS \\
0.006 \\
\hline
\end{tabular}

\begin{tabular}{ccc}
\cline { 1 - 1 } NS & & NS \\
NS & & NS \\
$<0.001$ & & NS \\
NS & & NS \\
NS & & NS \\
0.01 & & NS \\
NS & & NS \\
NS & & NS \\
NS & NS \\
\hline
\end{tabular}

${ }^{a} P$ value between $\mathrm{CHC}$ and POC users; ${ }^{b} P$ value between $\mathrm{CHC}$ and non-hormonal contraceptive users; ${ }^{c} P$ value between $\mathrm{POC}$ and non-hormonal contraceptive users.

AUC, area under the curve; CHCs, combined hormonal contraceptives; HOMA-IR, homeostatic model of assessment of insulin resistance; NS, nonsignificant; POCs, progestin-only contraceptives.

associated with preDM or T2DM by age 46. The factors significantly associated with hormonal contraception use at age 46 were included in multivariate binary logistic regression models. The results of the regression analyses are reported as odds ratios (ORs) with 95\% confidence intervals (95\% CIs). All analyses are reported as crude ORs and ORs adjusted for factors associated with the use of contraceptives and metabolic outcomes (i.e. socioeconomic status (SES), consumption of alcohol, smoking, parity, BMI, and use of cholesterol-lowering medication). IBM SPSS Statistics software version 22.0 for Windows was used for all statistical analyses. The level of statistical significance was set at $P \leq 0.05$.

\section{Results}

Waist circumferences and BMIs did not differ between $\mathrm{CHC}$, non-hormonal contraceptive, and POC users. CHC users had fewer deliveries than non-hormonal contraceptive users $(P<0.001)$ and POC users $(P<0.001)$. POC users had fewer deliveries than non-hormonal contraceptive users $(P=0.01)$ (Table 1$)$.

\section{Prevalence of glucose metabolism disorders}

Current $\mathrm{CHC}$ use was significantly associated with preDM (crude OR: 2.0, 95\% CI: 1.3-3.2) and T2DM (crude OR: 3.3, 95\% CI: 1.1-9.7) compared with non-hormonal contraceptive use. The use of $\mathrm{CHCs}$ for less than 5 years was not associated with disordered glucose metabolism compared with the use of non-hormonal contraceptives for less than 5 years. However, the use of CHCs for 5 years or more was associated with an increased risk of preDM when compared with the use of non-hormonal contraceptives for over 5 years (preDM $20.7 \%$ vs $12.3 \%$, crude OR: 2.2, 95\% CI: 1.3-3.7). Furthermore, the use of $\mathrm{CHCs}$ for 5 years or more was associated with an increased risk of T2DM compared with the use of non-hormonal contraceptives (T2DM $4.5 \%$ vs $0.7 \%$, crude OR: $4.5,95 \%$ CI: 1.5-13.5) (Table 2).

A total of 2 (1.3\%) CHC users, 16 (2.2\%) POC users, and $24(2.7 \%)$ non-hormonal contraceptive users were currently using statins at age 46. As statins may alter glucose metabolism, we performed sub-analyses that excluded statin users, and the risk of preDM or T2DM did not change in any of the groups studied. As for other risk factors for abnormal glucose tolerance, previous gestational diabetes mellitus (GDM) was diagnosed at a similar rate in all study groups: in 9 (5.9\%) CHC users, in $71(8.4 \%)$ POC users, and in 69 (7.8\%) non-hormonal contraceptive users. Moreover, the family history of T2DM (grandparents, parents, siblings and children) was asked as a part of the questionnaire in the clinical examinations. In all study groups, $94-96 \%$ answered this question. Among the CHC users, $32 \%$ had a family history of T2DM which was lower $(P=0.001)$ than in the POC $(46 \%)$ or in the non-hormonal contraceptive (48\%) user group.

In a subgroup analysis, we also investigated a separate group of women who reported $\mathrm{CHC}$ to use both at age 31 and at age $46(n=91)$. The analysis revealed that these women had a two-fold increased risk for preDM (crude OR: 2.0, 95\% CI: 1.0-3.7) but not a significant risk for T2DM compared with non-hormonal contraceptive use at age 46 .

In the entire study population, $\mathrm{CHC}$ use was significantly associated with preDM (crude OR: 1.9, 95\% CI: $1.2-3.0$, adjusted OR: $1.8,95 \%$ CI: $1.1-3.1)$ and T2DM (crude OR: 2.4, 95\% CI: 0.8-6.7, adjusted OR: 4.1, 95\% 
Table 2 The association of current CHC use with preDM and T2DM compared with current non-hormonal contraceptive use.

\begin{tabular}{l} 
Duration of use \\
\hline$n$ \\
PreDM, $n(\%)$ \\
$\quad$ Crude OR $(95 \% \mathrm{CI})$ \\
$\quad$ Adjusted ${ }^{\mathrm{a}}$ OR $(95 \% \mathrm{CI})$ \\
T2DM, $n(\%)$ \\
$\quad$ Crude OR $(95 \% \mathrm{Cl})$ \\
$\quad$ Adjusted ${ }^{\mathrm{O}}$ OR $(95 \% \mathrm{CI})$ \\
\hline
\end{tabular}

\begin{tabular}{c}
\hline Current use \\
\hline $153 / 884^{\mathrm{b}}$ \\
$29(19.0 \%)$ \\
$2.0(1.3-3.2)$ \\
$2.3(1.3-4.0)$ \\
$5(3.3 \%)$ \\
$3.3(1.1-9.7)$ \\
$5.3(1.6-18.2)$ \\
\hline
\end{tabular}

\begin{tabular}{c}
\hline$<\mathbf{5}$ years \\
\hline $32 / 89$ \\
$5(15.6 \%)$ vs $10(11.2 \%)$ \\
$1.4(0.4-4.6)$ \\
$0.98(0.2-3.9)$ \\
0 vs $1(1.1 \%)$ \\
\end{tabular}

\begin{tabular}{c}
\hline $\mathbf{5}$ years \\
\hline $111 / 423$ \\
$23(20.7 \%)$ vs $52(12.3 \%)$ \\
$2.2(1.3-3.7)$ \\
$2.3(1.3-4.4)$ \\
$25(4.5 \%)$ vs $3(0.7 \%)$ \\
$4.5(1.5-13.5)$ \\
$6.7(2.0-22.6)$
\end{tabular}

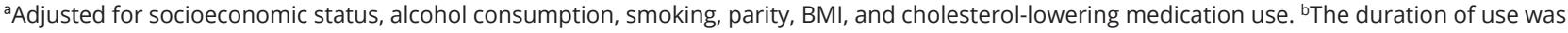
reported by 143 of 153 current $\mathrm{CHC}$ users and 512 of 884 current non-hormonal contraceptive users.

CHC, combined hormonal contraceptive; PreDM, prediabetes; T2DM, type 2 diabetes.

CI: 1.3-13.2) compared with POC use. The use of POCs was not associated with preDM or T2DM when compared with the use of non-hormonal contraceptives (data not shown).

\section{Glucose metabolism according to OGTTs}

CHC users had higher glucose AUC values $(P=0.01)$ in OGTT than non-hormonal contraceptive users. CHC users had higher glucose-AUC values $(P=0.001)$ and lower Matsuda Index values $(P=0.006)$ than POC users. The results remained similar after adjustments for BMI and waist circumference (Table 1).

\section{Discussion}

This population-based study shows that current use of $\mathrm{CHCs}$ at age 46 was associated with an increased risk for glucose metabolism disorders measured by OGTT. The sub-analysis revealed that the use of CHCs for 5 years or more increased the risk for preDM and T2DM. The current use of POCs was not associated with an increased risk for preDM or T2DM. Although overweight and obesity are known risk factors for glucose metabolism disorders, they did not explain our observations. Also, the previously diagnosed GDM or family history of T2DM did not explain the differences between the study groups.

The present results are consistent with our previous findings in the same cohort population, which showed decreased insulin sensitivity and higher levels of insulin at age 31 in women using CHCs despite lower BMIs in this group (8). The present study shows that some of the current CHC users developed preDM. This observation is in line with the results of a recent Swedish prospective population-based study that included 4794 women aged 36-56 and showed that current CHC use was associated with a four-fold risk of preDM and a seven-fold risk of IGT
(20). In addition, a case-control study conducted among Chinese women over 40 years of age demonstrated a 2.1fold overall risk of T2DM in current premenopausal $\mathrm{CHC}$ users, and the risk became significant as early as 1 year after use (19).

In the present study, a current $\mathrm{CHC}$ use duration of 5 years or more was associated with an increased risk of T2DM compared with non-hormonal contraception use of the same duration, although the clinical value of this finding must be treated with caution due to the low number of diagnosed T2DM cases. CHC use of less than 5 years was not associated with any glucose metabolism disorders. The role of the duration of CHC use remains controversial, as some studies have shown no significant correlation between duration and glucose metabolism disorders (25, 26), whereas others have suggested a tendency toward an increased risk of diabetes with longer CHC use (19). Variation in study design, hormonal contraceptive preparation, BMI, and ethnicity and inadequate sample sizes in some studies may explain the differences between studies (11). In the present study, the 91 women who reported $\mathrm{CHC}$ use at the ages of both 31 and 46 years showed a two-fold increased risk of preDM but not T2DM compared with those who reported non-hormonal contraceptive use at age 46 . This finding may be related to the relatively few women using CHCs at both time points, and follow-up of this particular group of women should reveal whether or not these metabolic findings persist and whether the risk of T2DM actually increases compared with that in women using non-hormonal contraceptives or POC. However, the expert panel of the American Diabetes Association (ADA) announced that eventually, up to $70 \%$ of people with preDM will develop type 2 diabetes (27). In addition, the Diabetes Prevention Program (DPP) revealed the annualized incidence rate of type 2 diabetes 11\% among patients with preDM (28).

Current CHC users exhibited higher glucose-AUCs and lower Matsuda Index values (i.e. they displayed 
decreased glucose tolerance and insulin sensitivity with compensatory insulin secretion) compared with current POC or non-hormonal contraceptive users. Similarly, earlier studies have shown higher 2-h glucose and fasting insulin levels in $\mathrm{CHC}$ users of childbearing age $(7,9)$. Our results fit well with the findings of our previous Finnish randomized, open-label study, which revealed worsened insulin sensitivity during $\mathrm{CHC}$ use among healthy, normal-weight women under 33 years of age (6). Similarly, the results of an Italian study showed decreased insulin sensitivity in 30 healthy, lean CHC users (9). The aforementioned and present findings suggest that many $\mathrm{CHC}$ users have decreased glucose tolerance, which results in compensatory increased insulin secretion.

CHCs may affect glucose metabolisms by several mechanisms. Reduced glucose tolerance has been linked to hormonal contraceptives containing high-dose EE and androgenic progestins (5). Earlier studies suggest that preparations containing natural estradiol could have a milder effect on glucose tolerance than those containing EE $(29,30,31)$. Although estrogen is thought to have an independent role, progestin component may also modify the action of estrogen for instance by altering insulin response to glucose $(32,33)$. In this study, the data on the composition of different $\mathrm{CHC}$ preparations were not available, and therefore, we could not compare the risk of glucose metabolism alterations between different $\mathrm{CHC}$ generations, progestins or difference between $\mathrm{EE}$ and estradiol. Previous studies, however, have suggested that newer progestins, such as drospirenone and dienogest, may have less effects on glucose metabolism $(29,34)$.

In the present study, current POC use was not associated with preDM and/or T2DM compared with nonhormonal contraceptive use. This observation supports the results of earlier studies that showed that POCs have no effect (18) or only mild and clinically non-significant effects on insulin sensitivity $(12,13,15)$. All these studies suggest that POCs have minimal influence on glucose metabolism and may be safer contraceptives than CHCs in regard to T2DM risk. Most studies on the associations between hormonal contraception and alterations of glucose metabolism have mainly involved young, healthy, and non-obese women. Therefore, as overweight status is becoming more common worldwide, more attention should be directed toward middle-aged women who start to display unfavorable alterations in body weight, blood pressure, lipid profiles, and glucose metabolism, which together with physical inactivity contribute to an increased risk and incidence of cardiovascular diseases $(28,35,36)$. The present results suggest that POCs should be preferred to CHCs as contraception for women with increased metabolic risk factors.

\section{Strengths and limitations}

The greatest strength of this study resides in the characteristics of the study population; the NFBC1966 data set provided a unique opportunity to investigate the association between the use of hormonal contraceptives and glucose metabolism disorders in a large non-selected population of perimenopausal women. In addition, the long-term follow-up of the same population allowed us to compare observations at the ages of 31 and 46. Our results are based on OGTTs, which were performed in all our study participants. We were also able to include several confounding factors in the analysis. There are also limitations, including the fact that the data on the current use of hormonal and non-hormonal contraceptives were based on self-reporting. However, the responses were confirmed by another questionnaire during the clinical examinations.

Ninety-four percent of $\mathrm{CHC}$ and $98 \%$ of POC users reported the length of use of hormonal preparations whereas only $58 \%$ of non-hormonal contraceptive users reported the length of use of non-hormonal contraception. A previous use of $\mathrm{CHC}$ among nonhormonal contraception users could have biased our results, as a Swedish study found an association between both current and previous use of $\mathrm{CHC}$ and prediabetes. The risk was four times higher in current and two times higher in former users at the beginning of the study when compared to women who had never used CHC. During the 8 years' follow-up, however, previous $\mathrm{CHC}$ use did not confer increased risk for abnormal glucose tolerance, and the conclusion was that the possible association between CHC use and abnormal glucose metabolism is transient (20). Likewise, a Chinese study reported that the risk of T2DM decreased after cessation of CHC use in a timedependent manner (19). Last, the Nurses Health Study found a marginally increased risk of T2DM in previous CHC users (RR: 1.10, 95\% CI: 1.01-1.21), who had stopped the use at least 5 years earlier, compared with women who had never used CHC (37). Given all this and the fact that in the present study $83 \%$ of non-hormonal contraception users had not used any hormonal contraception for the last 5 years minimum, the possibility of $\mathrm{CHC}$ affecting glucose tolerance in the non-hormonal contraception users is unlike.

Lastly, women with pre-existing risk factors for impaired glucose metabolism were probably less likely to 
use CHCs at perimenopausal age, and therefore, the use of $\mathrm{CHCs}$ may be associated with an even greater risk of glucose metabolism disorders in an unselected user population.

The present findings suggest an increased risk for impaired glucose metabolism in current CHC users of perimenopausal age. This finding raises the question of whether it is more appropriate to recommend POCs or nonhormonal contraceptives over CHCs to perimenopausal women with known metabolic risks. Further studies should also elaborate the difference between EE and estradiol as for their metabolic properties.

\section{Declaration of interest}

T $\mathrm{P}$ has receiver advisory board and lecturing honorarium from Exeltis, Merck, Ferring, and M S D. T P also participated in the E4 FREEDOM trial (NCT02817841) with PRA Health Sciences. These affiliations do not conflict with the present research. The remaining authors have nothing to disclose.

\section{Funding}

This study was supported by grants from the Sigrid Jusélius Foundation, the Academy of Finland, Oulu and Helsinki University Hospital Research Funds, and Oulu University Medical Research Center.

\section{References}

1 Wynn V \& Doar JW. Some effects of oral contraceptives on carbohydrate metabolism. Lancet 19662 715-719. (https://doi. org/10.1016/s0140-6736(66)92978-3)

2 Wynn V, Adams PW, Godsland I, Melrose J, Niththyananthan R, Oakley NW \& Seed M. Comparison of effects of different combined oral-contraceptive formulations on carbohydrate and lipid metabolism. Lancet 19791 1045-1049. (https://doi.org/10.1016/ s0140-6736(79)92949-0)

3 Wynn V \& Godsland I. Effects of oral contraceptives on carbohydrate metabolism. Journal of Reproductive Medicine 198631 (Supplement) 892-897. (https://doi.org/10.1136/jcp.s1-3.1.19)

4 Godsland IF, Crook D \& Wynn V. Low-dose oral contraceptives and carbohydrate metabolism. American Journal of Obstetrics and Gynecology 1990163 348-353. (https://doi.org/10.1016/00029378(90)90580-z)

5 Sitruk-Ware R \& Nath A. Characteristics and metabolic effects of estrogen and progestins contained in oral contraceptive pills. Best Practice and Research: Clinical Endocrinology and Metabolism 201327 13-24. (https://doi.org/10.1016/j.beem.2012.09.004)

6 Piltonen T, Puurunen J, Hedberg P, Ruokonen A, Mutt SJ, Herzig KH, Nissinen A, Morin-Papunen L \& Tapanainen JS. Oral, transdermal and vaginal combined contraceptives induce an increase in markers of chronic inflammation and impair insulin sensitivity in young healthy normal-weight women: a randomized study. Human Reproduction 2012 27 3046-3056. (https://doi.org/10.1093/humrep/des225)

7 Elkind-Hirsch KE, Darensbourg C, Ogden B, Ogden LF \& Hindelang P. Contraceptive vaginal ring use for women has less adverse metabolic effects than an oral contraceptive. Contraception 200776 348-356. (https://doi.org/10.1016/j.contraception.2007.08.001)

8 Morin-Papunen L, Martikainen H, McCarthy MI, Franks S, Sovio U, Hartikainen AL, Ruokonen A, Leinonen M, Laitinen J, Jarvelin MR et al. Comparison of metabolic and inflammatory outcomes in women who used oral contraceptives and the levonorgestrel- releasing intrauterine device in a general population. American Journal of Obstetrics and Gynecology 2008199 529.e1-529.e10. (https://doi.org/10.1016/j.ajog.2008.04.013)

9 Cagnacci A, Ferrari S, Tirelli A, Zanin R \& Volpe A. Route of administration of contraceptives containing desogestrel/ etonorgestrel and insulin sensitivity: a prospective randomized study. Contraception 200980 34-39. (https://doi.org/10.1016/j. contraception.2009.01.012)

10 Gaspard U, Scheen A, Endrikat J, Buicu C, Lefebvre P, Gerlinger C $\&$ Heithecker R. A randomized study over 13 cycles to assess the influence of oral contraceptives containing ethinylestradiol combined with drospirenone or desogestrel on carbohydrate metabolism. Contraception 200367 423-429. (https://doi. org/10.1016/s0010-7824(02)00537-1)

11 Lopez LM, Grimes DA \& Schulz KF. Steroidal contraceptives: effect on carbohydrate metabolism in women without diabetes mellitus. Cochrane Database of Systematic Reviews 20144 CD006133. (https:// doi.org/10.1002/14651858.CD006133.pub5)

12 Kahn HS, Curtis KM \& Marchbanks PA. Effects of injectable or implantable progestin-only contraceptives on insulin-glucose metabolism and diabetes risk. Diabetes Care 200326 216-225. (https://doi.org/10.2337/diacare.26.1.216)

13 Biswas A, Viegas OA, Coeling Bennink HJ, Korver T \& Ratnam SS. Implanon contraceptive implants: effects on carbohydrate metabolism. Contraception 200163 137-141. (https://doi. org/10.1016/s0010-7824(01)00182-2)

14 Dorflinger LJ. Metabolic effects of implantable steroid contraceptives for women. Contraception 200265 47-62. (https://doi.org/10.1016/ s0010-7824(01)00290-6)

15 Konje JC, Otolorin EO \& Ladipo OA. Changes in carbohydrate metabolism during 30 months on Norplant. Contraception 199144 163-172. (https://doi.org/10.1016/0010-7824(91)90116-w)

16 Segall-Gutierrez P, Du J, Niu C, Ge M, Tilley I, Mizrajj K \& Stanczyk FZ. Effect of subcutaneous depot-medroxyprogesterone acetate (DMPA-SC) on serum androgen markers in normal-weight, obese, and extremely obese women. Contraception 201286 739-745. (https://doi.org/10.1016/j.contraception.2012.05.148)

17 Bender NM, Segall-Gutierrez P, Najera SO, Stanczyk FZ, Montoro M $\&$ Mishell DR. Effects of progestin-only long-acting contraception on metabolic markers in obese women. Contraception 201388 418-425. (https://doi.org/10.1016/j.contraception.2012.12.007)

18 Koopersmith TB \& Lobo RA. Insulin sensitivity is unaltered by the use of the Norplant subdermal implant contraceptive. Contraception 199551 197-200. (https://doi.org/10.1016/0010-7824(95)00016-4)

19 Rosenthal AD, Shu XO, Jin F, Yang G, Elasy TA, Li Q, Xu HX, Gao YT \& Zheng W. Oral contraceptive use and risk of diabetes among Chinese women. Contraception 200469 251-257. (https://doi. org/10.1016/j.contraception.2003.10.015)

20 Deleskog A, Hilding A \& Ostenson CG. Oral contraceptive use and abnormal glucose regulation in Swedish middle aged women. Diabetes Research and Clinical Practice 201192 288-292. (https://doi. org/10.1016/j.diabres.2011.02.014)

21 Allen RH, Cwiak CA \& Kaunitz AM. Contraception in women over 40 years of age. Canadian Medical Association Journal 2013185 565-573. (https://doi.org/10.1503/cmaj.121280)

22 Baldwin MK \& Jensen JT. Contraception during the perimenopause. Maturitas 201376 235-242. (https://doi.org/10.1016/j. maturitas.2013.07.009)

23 Hanley AJ, McKeown-Eyssen G, Harris SB, Hegele RA, Wolever TM, Kwan J \& Zinman B. Association of parity with risk of type 2 diabetes and related metabolic disorders. Diabetes Care 200225 690-695. (https://doi.org/10.2337/diacare.25.4.690)

24 Shen L, Wu J, Xu G, Song L, Yang S, Yuan J, Liang Y \& Wang Y. Parity and risk of coronary heart disease in middle-aged and older Chinese women. Scientific Reports 20155 16834. (https://doi.org/10.1038/ srep16834) 
25 Troisi RJ, Cowie CC \& Harris MI. Oral contraceptive use and glucose metabolism in a national sample of women in the United States. American Journal of Obstetrics and Gynecology 2000183 389-395. (https://doi.org/10.1067/mob.2000.105909)

26 Furman BL. Impairment of glucose tolerance produced by diuretics and other drugs. Pharmacology and Therapeutics 198112 613-649. (https://doi.org/10.1016/0163-7258(81)90102-9)

27 Tabak AG, Herder C, Rathmann W, Brunner EJ \& Kivimaki M. PreDM: a high-risk state for diabetes development. Lancet 2012379 2279-2290. (https://doi.org/10.1016/S0140-6736(12)60283-9)

28 Godsland IF. Interaction of oral contraceptive use with the effects of age, exercise habits and other cardiovascular risk modifiers on metabolic risk markers. Contraception 199653 9-16. (https://doi. org/10.1016/0010-7824(95)00260-x)

29 De Leo V, Fruzzetti F, Musacchio MC, Scolaro V, Di Sabatino A \& Morgante G. Effect of a new oral contraceptine with estradiol valerate/dienogest on carbohydrate metabolism. Contraception 2013 88 364-368. (https://doi.org/10.1016/j.contraception.2012.09.003)

30 Junge W, Mellinger U, Parke S \& Serrani M. Metabolic and haemostatic effects of estradiol valerate/dienogest, a novel oral contraceptive: a randomized, open-label, single-centre study. Clinical Drug Investigation 201131 573-584. (https://doi. org/10.2165/11590220-000000000-00000)

31 Ågren UM, Anttila M, Mäenpää-Liukko K, Rantala ML, Rautiainen H, Sommer WF \& Mommers E. Effects of a monophasic combined oral contraceptive containing nomegestrol acetate and $17 \beta$-oestradiol in comparison to one containing levonorgestrel and ethinylestradiol on markers of endocrine function. European Journal of Contraception and Reproductive Health Care 201116 458-467. (https://doi.org/10.3109/1 3625187.2011.614363
32 Kojima T, Lindheim SR, Duffy DM, Vijod MA, Stanczyk FZ \& Lobo RA. Insulin sensitivity is decreased in normal women by doses of ethinyl estradiol used in oral contraceptives. American Journal of Obstetrics and Gynecology 1993169 1540-1544. (https://doi. org/10.1016/0002-9378(93)90432-i)

33 Godsland IF, Walton C, Felton C, Proudler A, Patel A \& Wynn V. Insulin resistance, secretion, and metabolism in users of oral contraceptives. Journal of Clinical Endocrinology and Metabolism 1992 74 64-70. (https://doi.org/10.1210/jcem.74.1.1530790)

34 Oelkers W, Foidart JM, Dombrovicz N, Welter A \& Heithecker R. Effects of a new oral contraceptive containing an antimineralocorticoid progestogen, drospirenone, on the reninaldosterone system, body-weigth, blood pressure, glucose tolerance, and lipid metabolism. Journal of Clinical Endocrinology and Metabolism 199580 1816-1821. (https://doi.org/10.1210/jcem.80.6.7775629)

35 Isomaa B, Almgren P, Tuomi T, Forsen B, Lahti K, Nissen M, Taskinen MR \& Groop L. Cardiovascular morbidity and mortality associated with the metabolic syndrome. Diabetes Care 200124 683-689. (https://doi.org/10.2337/diacare.24.4.683)

36 Diabetes Prevention Program Research Group, Knowler WC, Fowler SE, Hamman RF, Christophi CA, Hoffman HJ, Brenneman AT, Brown-Friday JO, Goldberg R, Venditti E et al. 10-year follow-up of diabetes incidence and weight loss in the diabetes prevention program outcomes study. Lancet 2009374 1677-1686. (https://doi. org/10.1016/S0140-6736(09)61457-4)

37 Rimm EB, Manson JE, Stampfer MJ, Colditz GA, Willett WC, Rosner B, Hennekens CH \& Speizer FE. Oral contraceptive use and the risk of type 2 (non-insulin-dependent) diabetes mellitus in a large prospective study of women. Diabetologia 199235 967-972. (https:// doi.org/10.1007/BF00401427)

Received 22 April 2020

Revised version received 30 August 2020

Accepted 28 September 2020 